\title{
Introduction to the special theme on Internet marketing
}

\author{
Christopher P. Holland • Kristin Diehl • \\ Andreas Herrmann
}

Published online: 21 August 2013

(C) Institute of Information Management, University of St. Gallen 2013

The call for papers identified a set of related Internet marketing themes that were intended to capture the key research topics in this new and rapidly evolving subject area. Three diverse papers have been accepted for a special collection of papers that focus on different aspects of Internet marketing strategy. Based on the thematic areas identified in the call for papers, the authors Corley, Jourdan and Ingram developed a framework to assess the current research landscape of published papers in the top IS and marketing journals. Such an assessment of research topics and themes, use of different methodologies and trends over time is an important activity because a review of this type gives academics an overview of current and historical research approaches, and also gives perspective on the relative scale of efforts in different thematic areas. It can also be used to identify key research articles in specific areas and, therefore, is of great value to young researchers in the identification of a research problem, in particular $\mathrm{PhD}$ researchers and academics new to the field. Another benefit of an overview of the published research is to identify possible gaps and omissions in the literature, as well as to recognize future research trends. The authors' evaluation of research literature provides fascinating insights into academic developments in the field of Internet marketing, which deserve further discussion.

An immediate observation that can be made is that IS research focuses on business related topics rather than technical ones, e.g. on business models, advertising and business

C. P. Holland $(\bowtie)$

University of Manchester, Manchester Business School,

Booth Street West, M15 6PB Manchester, UK

e-mail: chris.holland@mbs.ac.uk

K. Diehl

University of Southern California, Los Angeles, CA, USA

e-mail: diehl@marshall.usc.edu

A. Herrmann

University of St. Gallen, St. Gallen, Switzerland

e-mail: andreas.herrmann@unisg.ch

performance in an online environment. This conclusion may seem obvious to IS researchers, but it may not always be clear to colleagues from other business disciplines, such as strategy and marketing, that IS researchers engage in research that crosses business and technology boundaries. The assessment of research methodologies identifies that the most prevalent approach is formal theory and literature review, which accounted for one quarter of all research methodologies. The authors interpret this finding as being indicative of a relatively new topic. Another interpretation is that it reflects the emphasis of academics on theoretical discussion and rigour, possibly with less importance being attached to relevance. Although formal literature reviews may be relevant to business managers, it is more likely that literature reviews are aimed almost exclusively at the academic community. Practising managers and academics who are more interested in topical and relevant research that has practical benefits are more likely to gain insights from empirically focused research supported by research frameworks and theoretical underpinnings. It is therefore encouraging that original data analysis in the form of sample surveys and field work based on primary data accounted for about 40 percent of published research. Two important thematic gaps are identified by the authors: search strategies and web 2.0. Given the strategic and commercial importance of search strategies in consumer e-commerce, the paucity of research in this area is rather surprising. Of the 17 papers published on search, only four contained primary data: two survey papers, one field study and one field experiment. Therefore, the second paper of this special theme by Nottorf and Funk is a timely and important contribution.

Nottorf and Funk's research into paid search is an excellent example of field work using primary data and employing sophisticated modelling techniques to capture important and subtle differences in performance based on a detailed analysis of key words and consumer behaviour in a cross-sectional study. This type of research highlights the advantages of researchers getting close to the phenomena of interest in order 
to apply and test established theoretical concepts in an organizational/Internet market setting. The results demonstrate the importance of key differences between market sectors, and also show specific managerial implications for developing paid search strategies related to brand terms and generic search behaviour. One important idea that emerges from the study is the recommendation to managers that active experimentation is important in order to improve and optimize paid search campaigns, and to test the results from this analysis in other market sectors.

The third paper in the collection of research articles on Internet marketing is authored by Reichhart, Pescher and Spann. It evaluates the performance of a marketing campaign across two channels: email and text. A large-scale survey of this type, involving over 37,000 participants, is a good illustration of new kinds of research data increasingly becoming available to Internet researchers. The paper also demonstrates the potentials of applying established research frameworks and concepts, such as price elasticity and advertising performance metrics from economics and marketing, in a new context, i.e. for email and text coupons in an Internet marketing application.
Looking ahead, it is clear that there is enormous potential for IS researchers to make significant contributions to both business and technical research themes in the subject of Internet marketing. The continued growth in the use of web 2.0 and social networking is likely to receive much more attention from academic researchers and this will be reflected in a higher share of research publications. The topics of online business models and advertising are key research areas in strategy and marketing. This also represents an opportunity for IS researchers to publish in journals outside of the traditional IS sphere. Given the fact that most of the new developments and innovations in Internet marketing are created and implemented by business organizations, it is imperative that academic researchers continue to maintain the balance between theory and practice, and actively seek to produce research that is both rigorous and relevant and, therefore, is of interest to both, academics and managers. By conducting research of this kind, academics will continue to benefit from access to market data and cutting-edge practice, and managers will benefit commercially from free exchange of ideas and conceptual thinking with academic researchers. 